\title{
Impact of Educational Intervention in Knowledge of Infection Prevention and Control among healthcare professionals in Nepal
}

\author{
Sait Kumar Pradhan, MBBS, MD \\ Department of Physiology, Institute of Medicine, TU \\ saitpradhan@gmail.com \\ Sweta Shrestha, PharmD \\ Department of Pharmacy, KU \\ swetashres@gmail.com
}

\begin{abstract}
Background: Health care-associated infection (HCAl) affects the mortality and morbidity of inpatients worldwide. Nepal is a developing country in which HCAl pose a major problem in terms of patient safety. Improper and inadequate knowledge about HCAl among health care workers is responsible for majority of nosocomial infections resulting in prolonged illness. Thus this study was designed to assess the level of knowledge of infection control among nurses and pharmacists.
\end{abstract}

Methods: A pre and post interventional study was conducted in 22 participants using a self-administered questionnaire which included queries on hand hygiene strategies, cross contamination sources and aseptic services. Each correct answer was scored as 1 and an incorrect answer was scored as zero. The total score for each individual was calculated before and after the intervention which was compared using the Paired t test at 95\% confidence interval.

Result: $81.8 \%$ of the participants possessed a good knowledge on infection prevention and control practices which increased to $100 \%$ after intervention. The difference in knowledge score before and after the workshop was found to be statistically significant $(p=0.034)$. Previous training and duration of work experience was found to be correlated with the knowledge score whereas the other independent variables had no association with the knowledge score.

Conclusion: Educational intervention has a positive impact in 
the knowledge level of the healthcare professionals in infection and prevention control.

Keywords: Infection Prevention, Infection Control, Intervention, Knowledge

\section{Introduction}

According to World Health Organization (WHO) an infection is considered a HCAI if it is occurring in a patient during the process of care in a hospital or other healthcare facility which was not present or incubating at the time of admission, this includes infections acquired in the hospital but appearing after discharge, and also occupational infections among staff of the facility. [1] HCAls impacts both developed and developing countries affecting over 1.4 million people globally.[2] However, reports suggest HCAls to be preventable issues [3], hand hygiene being an efficacious preventive strategy. [2,4] Inspite of the relative ease of the procedure, reluctance and lack of adherence among health care providers has been found to be widely prevalent. $[3,5,6]$ Among the various factors contributing to this lack of compliance; lack of training and experience and lack of knowledge on the significance of hand hygiene are the indispensble ones. [5-7]

HCAls occurrence rate is reportedly higher in the developing nations than in the developed nations. [8] Nepal being a developing country situated in South Asia, confronts a high burden of HCAls. [9] presumably due to lack of national guidelines on infection control and the current scenario demands appropriate interventions to minimize this statistics [Priyamvada Paudyal]. Perpetual training to health care professionals with feedback has served as an effective tool in increasing hand hygiene adherence and infection control $[4,6]$.

A workshop was therefore conducted in the premises of Kathmandu University on "Infection Prevention and Control in hospitals". Experts on the relevant field were invited as resource persons and majority of the participants were nursing staffs from "Dhulikhel hospital", Kavre and "Scheer Memorial Hospital", Banepa. Remainder were the pharmacists either engaged in hospitals or currently pursuing masters degree in Pharmaceutical Care. A total of 22 participants partook in the workshop. The experts discussed on the topics, namely IPAC (Infection Prevention and Control), Hand hygiene strategy and standard precautions and Aseptic services: Role of pharmacists and nurses. The present workshop on "Infection Prevention and Control in hospitals" was organized as an interventional measure with the objective of studying the knowledge of healthcare associated infection control among the participants before and after the training.

\section{Literature Review}

A study evaluating the knowledge, attitudes, and behaviour regarding hand hygiene of the physicians and nurses involved in intensive care units (ICUs) of five of the randomly selected hospitals in Italy was carried out by CGA et al. This was a questionnaire based study in which correct answers in relation to knowledge was consented upon by $53.2 \%$ 
of the participants and significantly higher level of knowledge was found among the partakers in neonatal, medicine and surgery wards. [10]

Another study by Paudyal et al. assessed the knowledge, attitude and infection control practices among healthcare workers in Nepal. A questionnaire based survey that undertook the response of 158 doctors and 166 nurses found that the staffs involved had a good level of knowledge and positive attitude towards majority of the aspects of infection control. Profession, age and abroad study were found to be the strong predictors of knowledge, attitude and practice.[9]

A study by Gurung G. assessed the effectiveness of infection control training program among peons working at peripheral healthcare facilities in Nepal. This was an interventional study which included an extensive five days training and follow up assessment for 6-8 months. Final evaluation was done after completion of one year. A significant improvement in infection control practice was observed after the training.[11]

\section{Objectives}

The primary objective of the study was to assess the impact of educational intervention in the knowledge level of the healthcare professionals. The secondary objectives were to determine the correlation between the independent variables and the knowledge score.

\section{Methodology}

The workshop was conducted on May 2016 in the premises of Kathmandu University, Nepal. At the beginning of the session, the participants were allowed to fill and submit a questionnaire on IPAC. Written informed consent form was obtained from all the participants for the study purpose beforehand. At the end of the workshop, the participants were requested to fill in the same questionnaire. The questionnaire was prepared by the researchers in consensus with the experts in the relevant field. A combination of True/ False statements, Yes/No questions and choosing the right answer was included in the questionnaire. The questionnaire comprised queries on hand hygiene strategies, cross contamination sources and aseptic services and was analyzed for ease of comprehension by two nursing professionals not involved in the study. Each correct statement or answer was allocated 1 point whereas an incorrect answer earned 0 point. The total score for each individual participant was calculated at two time points, namely before and after the workshop. Participants who got more than $50 \%$ of the questions correctly answered $(n \geq 11)$ was said to have "Good Knowledge" whereas those scoring less $(n<11)$ was classed as having "Poor Knowledge". All the data was entered and analyzed using Microsoft Excel. The scores before and after the workshop were compared using paired samples $t$-test. A p value less than 0.05 was taken as statistically significant. The association of sociodemographic variables with the knowledge score was analyzed using Pearson chi square test and Fischer exact test where applicable. 


\section{Results}

Majority of the participants (68.2\%) were of the age group less than 29 years. Female participants were greater in number (81.8\%) than male participants and there was equal participation of pharmacists and nursing personnel (50\%, 50\%). Approximately $68.2 \%$ of the partakers had completed their bachelors' level education whereas $13.6 \%$ were enrolled in Masters' degree program and only $18.2 \%$ had completed their PCL (Proficiency certificate level) in nursing program. Most of the participants $(63.6 \%)$ were working in hospitals, $22.7 \%$ were students, $9.1 \%$ were instructors in nursing colleges and $4.5 \%$ had their working experience in community pharmacy. A greater number of participants (77\%) reported of having received training on Infection prevention and control in the last 3 years whereas $23 \%$ had not received any form of formal training 3 years back.

The pretest status showed $81.8 \%$ of the participants possessed a good knowledge on infection prevention and control practices which rose to a figure of $100 \%$ after intervention. The difference in knowledge score before and after the workshop was found to be statistically significant $(p=0.034)$ using the paired $t$ test at $95 \%$ confidence interval. No significant association was seen between age, education, workplace and knowledge status, the likelihood ratio being $0.801,0.509,0.49$ respectively. Similarly gender and profession was found to be independent of the knowledge score (Fischer exact test $>0.05$ ) whereas significant association was observed between duration of work experience and knowledge score (Likelihood ratio $=0.021$ ). Receipt of training was also found to be correlated with the knowledge score obtained (Fischer exact test $<0.05$ ).

\section{Discussion}

Hand hygiene has proved to be an effective tool in preventing cross infection among patients in the hospitals. As per WHO, knowledge of hand hygiene practice is important in reducing HCAls. [12] Hence this study was carried out with the aim of assessing and improving the knowledge of healthcare professionals on hand hygiene strategies, cross infection sources and aseptic services.

Majority of the participants in our study had a good level of knowledge in the aforementioned criteria. This was probably due to the fact that most of the partakers were the nurses from Dhulikhel Hospital which has an effectively functioning IPAC committee that provides the essential information and trainings to its staff members. This figure is higher than similar survey from Italy (53\%) [10] and the study conducted by Paudyal et.al in Nepal (16\%). [9] The findings of this study is an addition to the fact that healthcare professionals in the capital city of Kathmandu possess a higher level of knowledge in IPAC than nurses in the western development region of Nepal (50.5\%). [8] This study also measured the effectiveness of the training on the knowledge level of the participants and a statistically significant difference was seen before and after the intervention. In a similar vein, a study conducted by Care Nepal which focused on educating and training the helpers at peripheral health care facilities in Nepal also showed that the infection control practice improved significantly after the intervention. [13] A contradictory result 
was observed in a study conducted by U Maharjan and S Mathew where no significant improvement in hand hygiene compliance was seen among the healthcare professionals one week and one month after the intervention. [14]

In regard to receipt of previous trainings, $77 \%$ had received some form of trainings on infection prevention and control. This data is similar to the findings of khanal $\mathrm{G}$ and Thapa S. where the percentage of prior training recipients was $74 \%$. [15] but much lower as compared to the data reported by Anargh et al. (91\%). [16] Duration of work experience and receipt of previous training were found to be significantly associated with the knowledge score which is in agreement with the findings by Asadollahi et al. [17]

\section{Conclusion}

Majority of the participants in the study had a good knowledge on infection prevention and control. A well designed training via the experts can make a significant positive impact in the knowledge level of the healthcare professionals. However, this was small scale study conducted only on nurses and pharmacists of limited hospitals. Further studies encompassing larger sample size and looking over the practice should be done.

\section{References}

A Guide to the Implementation of the WHO Multimodal Hand Hygiene Improvement

Strategy. World Health Organization available at https://www.who.int/gpsc/5may/ tools/who_guidelines-handhygiene_summary.pdf

Ansari SK, Gupta P, Jais M, Nangia S, Gogoi S, Satia S, et al. Assessment of the Knowledge, Attitude and Practices Regarding Hand Hygiene amongst the Healthcare Workers in a Tertiary Health Care Centre. Int J Pharma Res Health Sci. 2018;3(3):720-6.

Uneke $\mathrm{CJ}$ et al. Promotion of hand hygiene strengthening initiative in a Nigerian teaching hospital: implication for improved patient safety in low-income health facilities. Braz J Infect Dis. 201 4;1 8(1):21-27

Arthi $\mathrm{E}$ et al. Assessment of Knowledge, Attitude and Practice of Hand Hygiene among Nursing and Medical Students in a Tertiary Care Hospital in Puducherry, India. IJCMR. 2016; 3(4):1203-1206.

$\mathrm{K}$. Sodhi et al. Knowledge of infection control practices among intensive care nurses in a tertiary care hospital. J Infect Public Health. (2013) 6, 269-275

Teker B et al. Factors Affecting Hand Hygiene Adherence at a Private Hospital in Turkey. Eurasian J Med 2015; 47: 208-12.

Abdella et al. Hand hygiene compliance and associated factors among health care providers in Gondar University Hospital, Gondar, North West Ethiopia BMC Public Health 2014, 14:96.

Kim EG, Jeong I andThanju S. Knowledge, Attitude, and Compliance of Healthcareassociated Infection Control among Nurses in the Western Development Region, Nepal. Korean J Nosocomial Infect Control 2016; 21(1):9-17.I

Paudyal P, Simkhada P, Bruce J. Infection control knowledge, attitude, and practice among 
Nepalese health care workers. Am J Infect Control 2008;36:595-7.

World Health Organizaticon. (2002). Prevention of hospital-acquired infections: A practical guide. Retrieved from http://www.who.int/csr/resources/publications/ whocdscsreph200212.pdf (WHO 2002)

Nobile C, Diac E, Mantuori P, Villari P. Healthcare personnel and hand washing. Decontamination in intensive care units: knowledge, attitude and behavior in Italy. J Hosp Infect 2002; 51(3):226-232

Gurung G. Education and Training of Peon in Infection Control: Experience in Peripheral Health Facilities I Nepal. Int J Infect Control. 2009;5(1):1-5.

U Maharjan and S Mathew. A Study on Hand Hygiene Compliance among Health Care Professionals in ICU of a Selected Hospital. JCMC. 2013;3(5):4-9.

Khanal G and Thapa S. Awareness of Hand Hygiene Among Health Care Workers of Chitwan, Nepal. Sage Open. 2017:1-7.

Anargh, V., Singh, H., Kulkarni, A., Katwal, A., \& Mahen, A. HH practices among health care workers (HCWs) in a tertiary care facility in Pune. Medical Journal Armed Forces India.2013; 69, 54-56.

Asadollahi et al. Nurses' Knowledge Regarding Hand Hygiene and Its Individual and Organizational Predictors. J Caring Sci. 2015 Mar; 4(1): 45-53 